\title{
Localization of AQP5 during development of the mouse submandibular salivary gland
}

\author{
Helga S. Larsen - Marit H. Aure - Sarah B. Peters • \\ Melinda Larsen • Edward B. Messelt • \\ Hilde Kanli Galtung
}

Received: 26 October 2010/Accepted: 21 December 2010/Published online: 4 January 2011

(C) The Author(s) 2010. This article is published with open access at Springerlink.com

\begin{abstract}
Aquaporin 5 (AQP5) is known to be central for salivary fluid secretion. A study of the temporal-spatial distribution of AQP5 during submandibular gland (SMG) development and in adult tissues might offer further clues to its unknown role during development. In the present work, SMGs from embryonic day (E) 14.5-18.5 and postnatal days $(\mathrm{P}) 0,2,5,25$, and 60 were immunostained for AQP5 and analyzed using light microscopy. Additional confocal and transmission electron microscopy were performed on P60 glands. Our results show that AQP5 expression first occurs in a scattered pattern in the late canalicular stage and becomes more prominent and organized in the terminal tubuli/pro-acinar cells towards birth. Additional apical membrane staining in the entire intralobular duct is found just prior to birth. During postnatal development, AQP5 is expressed in both the luminal and lateral membrane of pro-acinar/acinar cells. AQP5 is also detected in the basal membrane of acinar cells at P25 and P60. In the intercalated ducts at P60, the male glands show apical staining in the entire segment, while only the proximal region is positive in the female glands. These results demonstrate an evolving distribution of AQP5 during pre- and postnatal development in the mouse SMGs.
\end{abstract}

Helga S. Larsen and Marit H. Aure contributed equally to this work.

H. S. Larsen $(\varangle) \cdot$ M. H. Aure · E. B. Messelt .

H. Kanli Galtung

Department of Oral Biology, Faculty of Dentistry,

University of Oslo, Sognsvannsveien 10, Rikshospitalet,

Blindern, Post Box 1052, 0316 Oslo, Norway

e-mail: h.s.larsen@odont.uio.no

S. B. Peters - M. Larsen

Department of Biological Sciences, University at Albany,

State University of New York, Albany, NY, USA
Keywords Submandibular gland - AQP5 - Development . Prenatal $\cdot$ Postnatal $\cdot$ Sexual dimorphism

\section{Introduction}

Aquaporin 5 (AQP5) belongs to a family of water channel proteins that allow water to pass through the plasma membrane by osmosis (Ishibashi et al. 2009; Krane and Goldstein 2007). This aquaporin has previously been detected in acinar cells of adult humans, mice, and rats (Akamatsu et al. 2003; Gresz et al. 2001; Ma et al. 1999), and its mRNA and protein have also been found to be expressed in the mouse submandibular salivary gland (SMG) during embryonic (Abate et al. 2003; Walker et al. 2008; Wei et al. 2007) and postnatal development (Larsen et al. 2009). Although several aquaporins (AQPs) are present in the mouse SMG (Larsen et al. 2009, 2010), studies with $\mathrm{AQP5}{ }^{-1-}$ mice have demonstrated a key role for AQP5 in salivary fluid secretion (Krane et al. 2001; Ma et al. 1999). However, its temporal-spatial distribution during development is not well documented. A detailed characterization is, therefore, needed to work towards an understanding of the role of this important aquaporin, frequently used as an acinar marker, during development. Also, a systematic knowledge of changes in AQP5 expression and localization could reduce misinterpretations when comparing published results from various developmental stages.

On the basis of structure, prenatal development of the SMG can be roughly divided into several stages. Briefly, from an initial bud, the gland undergoes branching morphogenesis and reaches the pseudoglandular stage ( $\sim$ embryonic day 14.5; E14.5) as a solid stalk having terminal end buds. In the following early and late canalicular ( $\sim$ E14.5-E16.5) and early and late terminal bud 
( $\sim$ E16.5-18.5) stages, lumens start to develop in the ductal segments and by birth a continuous lumen is present (see (Jaskoll and Melnick 1999; Tucker 2007) for further details). The SMG continues its development postnatally and by postnatal day 25 (P25) (young adults), the secretory units consist of seromucous cells (Young and Van Lennep 1978). The intralobular duct segments found most proximal to these units are intercalated ducts (ID), followed by granular convoluted tubules (GCT), and striated ducts (SD). At this point, sexual dimorphism is evident in the mouse SMG (Gresik and Macrae 1975). In general, morphological gender differences in adult glands are primarily manifested by a higher percentage of prominently larger GCT cells and smaller acinar cells in the male as compared to the female gland (Chai et al. 1993).

This study describes in detail the AQP5 localization during prenatal development as well as selected postnatal ages of the mouse SMG. A combination of three immunohistochemical techniques was used to investigate the expression pattern of AQP5 in the adult mouse SMG. In the prenatal gland we first detect AQP5 in a scattered pattern in the terminal bud cells. Towards birth, AQP5 is found in all terminal bud cells and adjacent ductal cells. In addition, during the last stage of prenatal development, AQP5 is expressed in the entire intralobular duct (IAD). In acinar cells of adult animals, AQP5 is localized to luminal, lateral, and basal membranes of acinar cells of both sexes, whereas we find a gender-specific expression pattern for AQP5 in the intercalated duct of adult glands at P60.

\section{Materials and methods}

Animals

CD-1 mice were treated according to the Norwegian Animal Welfare Act and the Regulation of Animal Experiments. The mice were sacrificed by cervical dislocation (adult mice) or decapitation (embryonic and newborn stages). Mice from E14.5-18.5 (from pseudoglandular stage to terminal bud stage) and P0, 2, 5, 25, and 60 were used. The day of plug discovery was designated as day 0.5 . Due to the lack of morphological sexual dimorphism present in the mouse SMG during prenatal (E14.5-18.5) and early postnatal development (P0, P2, P5), investigations of segregated sexes were only performed on young adults (P25) and adults (P60). Three SMGs from each time point were harvested from three independent litters/adult mice.

\section{Antibodies}

The same primary AQP5 antibody (\#AQP-005, Alomone Laboratories, Israel) was used in light $(0.06 \mu \mathrm{g} / \mathrm{ml})$, confocal $(1.5 \mu \mathrm{g} / \mathrm{ml})$, and electron microscopy $(20 \mu \mathrm{g} / \mathrm{ml})$. Other primary antibodies used in confocal microscopy recognized AQP5 (AB3559, Millipore, $0.4 \mathrm{mg} / \mathrm{ml}$ ), total collagen type IV (coll IV, AB769, Millipore, $4 \mu \mathrm{g} / \mathrm{ml}$ ), E-cadherin (clone 36, BD Transduction, $1 \mu \mathrm{g} / \mathrm{ml}$ ), and Zonula occluden-1 (ZO1) (clone ZO1-1A12, Invitrogen, $5 \mu \mathrm{g} / \mathrm{ml})$. The secondary antibody used for light microscopy was HRP-conjugated OMap anti-Rb secondary antibody (Ventana Medical Systems, Illkirch CEDEX, France). For confocal microscopy Cyanine dye-conjugated AffiniPure $\left.\mathrm{F}(\mathrm{ab})_{2}\right)_{2}$ antibodies were used (Jackson ImmunoResearch Laboratories $24 \mu \mathrm{g} / \mathrm{ml}$ ): Cy 2 anti-mouse for E-cadherin, Cy3 anti-rabbit for AQP5, Cy5 anti-goat for collagen IV, and Cy2 anti-mouse for ZO1. The cognate peptide for AQP5 was from Alomone Laboratories. Isotyped matched immunoglobulin $\mathrm{G}$ (IgG) control from Santa Cruz Biotechnology (CA, USA) was used in light $(1 \mu \mathrm{g} / \mathrm{ml})$ and electron microscopy $(20 \mu \mathrm{g} / \mathrm{ml})$.

\section{Immunohistochemistry}

The submandibular salivary glands were isolated, transferred to $10 \%$ NBF (neutral buffered formalin, SigmaAldrich Corp., St. Louis, MO, USA), and kept in this solution over night at $4^{\circ} \mathrm{C}$. After $24 \mathrm{~h}$, glands were placed in $70 \%$ ethanol and kept at $4{ }^{\circ} \mathrm{C}$ until the day of embedding. On the day of embedding, the tissue was dehydrated in a graded series of ethanol $(70,96,100 \%)$ and then transferred to xylene at room temperature $\left(20^{\circ} \mathrm{C}\right)$. Following paraffin infiltration for $1 \mathrm{~h}$ at $60^{\circ} \mathrm{C}$, the tissue was paraffin embedded. Serial sections $(6 \mu \mathrm{m})$ of entire glands (E13.5E18.5, P0, P2, and P5) or parts of glands (P25 and P60), were cut using a rotary microtome (Leica Microsystems, Wetzlar, Germany). As many sections as possible were placed on SuperFrost ${ }^{\circledR}$ Plus glass slides (Menzel-Gläser, Braunschweig, Germany), and each glass slide (containing material from one gland) was dried for $2 \mathrm{~h}$ at $60^{\circ} \mathrm{C}$ prior to immunohistochemical staining. Automated staining was performed using a Discovery XT (Ventana Medical Systems, Illkirch CEDEX, France) and the Research IHC Omni-UltraMap HRP XT procedure (Ventana Medical Systems, Illkirch CEDEX, France). Briefly, the sections were preconditioned using a Tris/Borate/EDTA based buffer ( $\mathrm{pH} 8,100^{\circ} \mathrm{C}$, Ventana) before being incubated in primary AQP5 antibody for $60 \mathrm{~min}$. The slides were then incubated in a HRP-conjugated OMap anti-Rb secondary antibody for $16 \mathrm{~min}$, followed by an $8 \mathrm{~min}$ incubation in 3,3'-diaminobenzidine (DAB) and hematoxylin counterstaining. The slides were rinsed in Reaction Buffer (Ventana Medical Systems, Illkirch CEDEX, France) between each step. After the automated staining, the slides were thoroughly rinsed in water containing detergent to remove any oily residues. The slides were then dehydrated in 
ethanol $(70,96,100 \%)$ and xylene before coverslip mounting. Negative controls were performed by incubating the antibody with tissue known to contain several other AQPs but not AQP5 (E18.5 kidney) (Parreira et al. 2009) and by using an isotyped matched $\mathrm{IgG}$ in place of the primary antibody. Sections were examined with a Nikon Eclipse 800 microscope (Nikon Instrument Inc., Melville, New York, USA) and photographed with a Nikon DS-5M camera (Melville, New York).

\section{Confocal microscopy}

Submandibular salivary glands were harvested and fixed in $10 \% \mathrm{NBF}$, as described above, and stored at $4^{\circ} \mathrm{C}$ in $70 \%$ ethanol prior to performing whole mount immunocytochemistry. Prior to immunocytochemistry, the glands were sliced into thick sections approximately $500 \mu \mathrm{m}$ in diameter and $50 \mu \mathrm{m}$ thick to improve antibody penetration. These slices were then rehydrated by rinsing for $10 \mathrm{~min}$ in 50 and $25 \%$ ethanol followed by $1 \times$ PBS. Immunostaining was performed as previously described (Daley et al. 2009; Larsen et al. 2003). Briefly, tissues were incubated in blocking solution (20\% donkey serum (Jackson ImmunoResearch) containing Mouse on Mouse (M.O.M.) blocking reagent (Vector Laboratories, Burlingame, CA, USA) in $1 \times$ PBS containing $0.05 \%$ Tween 20 (PBS-T)). Primary antibodies were diluted in an antibody diluent consisting of $10 \%$ bovine serum albumin (BSA, Sigma-Aldrich Corp., St. Louis, MO, USA) in phosphate-buffered saline (PBS) (PBSA) and incubated overnight at $4{ }^{\circ} \mathrm{C}$. Antibody dilutions used were: AQP5 (Alomone antibody; 1:300, Millipore antibody; 1:100), coll IV (1:100), E-cadherin (1:100), and ZO1 (1:100). SMG sections were washed $4 \times 10 \mathrm{~min}$ in $1 x$ PBS-T after each antibody incubation step. Cyanine dyeconjugated AffiniPure $\left.\mathrm{F}(\mathrm{ab})_{2}\right)_{2}$ fragment secondary antibodies were diluted 1:50 in diluent and incubated with the tissue overnight at $4^{\circ} \mathrm{C}$. Following the final wash sequence, tissues were mounted on glass coverslips with Secure-seal imaging spacers (Grace Bio-Labs) in $35 \mu \mathrm{l}$ FLUORO-GEL GelMount (Electron Microscopy Sciences) containing $1 \mathrm{mg} / \mathrm{mL}$ p-phenylene-diamine (PPD) antifade reagent. Three glands from three animals of both sexes were analyzed.

Tissues were imaged on a Zeiss LSM510 confocal microscope using argon $(488 \mathrm{~nm})$, HeNe1 $(543 \mathrm{~nm})$ and HeNe2 $(633 \mathrm{~nm})$ laser lines at $20 \times(\mathrm{Plan}$ Apo/0.75 NA) or $63 \times$ (Plan Apo/1.4 NA) magnification, with no bleed through between channels detected. All images were captured using identical settings. Negative controls included incubations with secondary antibody only, which showed no detectable signal, and peptide-preabsorbed negative control. The peptide-preabsorbed antibody control was prepared with a molar ratio of 10:1 (peptide: antibody) overnight at $4^{\circ} \mathrm{C}$. This solution was centrifuged at
$13,000 \mathrm{~g}$ for $30 \mathrm{~min}$ prior to addition of the supernatant to the tissues as a peptide-pre-absorbed control. The preabsorbed control supernatant was incubated overnight with the tissue at $4^{\circ} \mathrm{C}$ in parallel with the other samples.

Immunogold electron microscopy

Submandibular salivary glands from male and female animals (P60) were cut into pieces and fixed in $1 \%$ glutaraldehyde (Electron Microscopic Sciences, Industry Road, Hatfield, PA, USA) in Sörensen's phosphate buffer $\left(0.1 \mathrm{M}\right.$ solution of $\mathrm{NaH}_{2} \mathrm{PO}_{4}$ and $\left.\mathrm{Na}_{2} \mathrm{HPO}_{4}, \mathrm{pH} 7.2\right)$ (Prolabo chemicals, VWR International, West Chester, PA, USA) over night at $4^{\circ} \mathrm{C}$. After $24 \mathrm{~h}$, glands were placed in $0.5 \%$ glutaraldehyde and kept at $4^{\circ} \mathrm{C}$ until standard embedding in Lowicryl HM23 (Electron Microscopic Sciences, Industry Road Hatfield, PA, USA). Ultrathin sections $(70 \mathrm{~nm})$ of the glands were cut using a Leica ultracut UCT microtome (Leica Microsystems, Wetzlar, Germany), and the sections were placed on a 300 mesh nickel grid (Electron Microscopic Sciences Industry Road, Hatfield, PA, USA). The sections were blocked for nonspecific staining in 5\% BSA (BSA, Sigma-Aldrich Corp., St. Louis, MO, USA) in PBS for 120 min before incubation overnight with anti-AQP5 (Alomone labs). The sections were then washed in $1 \%$ PBSA $5 \times 5 \mathrm{~min}$ in room temperature before incubation with $10 \mathrm{~nm}$ colloidal gold conjugated with protein A in 1\% PBSA for 90 min (1:60, gift from Dr. Brorson, Institute of Pathology, University of Oslo, Norway). After washing in $1 \%$ PBSA $3 \times 5$ min followed by $4 \times 2$ min wash in ultrapure water, the sections were post-fixed in $2.5 \%$ glutaraldehyde/0.1 sodium cacodylat buffer (Fluka/Sigma-Aldrich Corp., St. Louis, MO, USA) for $2 \mathrm{~min}$ at room temperature. The sections were washed again in ultrapure water at room temperature $4 \times 2$ min before staining with $1 \%$ uranyl acetate (Electron Microscopic Sciences Industry Road, Hatfield, PA, USA) for $15 \mathrm{~min}$. The sections were rinsed with distilled water and further stained with $0.3 \%$ lead citrate (Fluka, Sigma-Aldrich Corp., St. Louis, MO, USA) for $3 \mathrm{~min}$ before the final rinse with distilled water. Negative controls were performed using IgG in place of primary antibody or by omitting the primary antibody. Three glands from three animals of both sexes were analyzed. The sections were examined using a CM 120 transmission electron microscope (Philips, Eindhoven, The Netherlands). Images were captured using a Morada camera on the iTEM platform (Soft Imaging System, Münster, Germany).

Image processing

Adobe Photoshop ${ }^{\circledR} \mathrm{CS} 2$ version 9.0.2. (Adobe Systems Incorporated, San Jose, CA) was used to perform image 
adjustments. Changes in brightness and contrast were applied to entire light microscopy pictures and levels (nonlinear) were adjusted for entire TEM images. No image processing was performed on the confocal images.

\section{Results}

Prenatal development

During prenatal development, SMG cells showed an increasing degree of AQP5 staining (Table 1). The glands do not uniformly mature, and we will therefore not refer to embryonic days but rather to developmental stages. At the pseudoglandular stage, no AQP5 was detected in the terminal bud or in the epithelial stalk (Fig. 1A). Similarly, in the early canalicular stage, the terminal bud and presumptive duct were also negative (Fig. 1B). In the late canalicular stage the first indication of AQP5 expression was detected in some cells in the terminal bud. All of the positive cells showed cytoplasmic staining, while some of them also had prominently positive plasma membranes. No AQP5 could be detected in the presumptive duct at this stage (Fig. 1C). In the early terminal bud stage, where the pro-acinar cells appeared to be more organized, all stained positive for AQP5 (Fig. 1D). Similar to the late canalicular stage, cytoplasmic staining could be observed, and apical and lateral membranes were prominently positive in all pro-acinar cells. In the IAD, the cells closest in proximity to the pro-acinar cells were also positive for AQP5, but only in the apical membrane. The rest of the IAD and the IED were negative (Fig. 1D). In the late terminal bud stage, where the pro-acinar cells are further organized, AQP5 was primarily detected in the apical and lateral membranes (Fig. 1E). The cytoplasmic staining appeared to be less prominent than in the previous stages, although there was some variability, which may be explained by pro-acinar cells within the same gland existing in multiple stages of maturation. The passage from the pro-acinar cells to the IAD cells was readily observed at this stage, and the entire IAD showed positive apical staining. However, the IED was still negative (Fig. 1F). The mesenchyme cells and the cells in the vascular system did not demonstrate detectable AQP5 staining throughout all prenatal stages studied.

\section{Postnatal development}

During postnatal development, the submandibular gland cells demonstrated a distinct pattern of AQP5 staining (Table 2). The postnatal development has not yet been classified as named stages and we will, therefore, refer to the specific postnatal days. Some studies have described two cell types in the secretory portion of the gland at birth (P0), terminal tubule cells and pro-acinar cells, based on intracellular features (Denny et al. 1988; Zinzen et al. 2004). In this study, we refer to them both as pro-acinar cells and all of them showed extensive apical and lateral staining at P0. A weaker staining was detected within the basal membrane and in the cytoplasm (Fig. 2A). The ductal segments adjacent to the pro-acinar cells had become closely packed with high-cuboidal cells with dense nuclei, and could be characterized as ID. AQP5 was present in the apical membranes of these cells (Fig. 2A). The rest of the IAD was negative, unlike the previous developmental stage (late terminal bud stage; right before birth). The remaining duct system was also negative (interlobular and main excretory duct). The AQP5 localization pattern at P2 was consistent with what found at P0 (data not shown). Five days after birth (P5), the pro-acinar cells exhibited a similar staining pattern as $\mathrm{P} 0$ and $\mathrm{P} 2$. Apical staining could also be observed in the ID segment (Fig. 2B). At this stage, the remaining ductal segment within one lobe, referred to as the SD, consisted of one layer of cuboidal cells and was negative for AQP5. The remaining ductal segments outside the lobes consist of both interlobular and main excretory ducts, and all were negative for AQP5. In young adults

Table 1 AQP5 localization during prenatal development

\begin{tabular}{|c|c|c|c|c|c|}
\hline & \multirow{2}{*}{$\begin{array}{l}\text { Pseudoglandular } \\
\text { stage }(\sim \mathrm{E} 14)\end{array}$} & \multicolumn{2}{|c|}{ Canalicular stage $(\sim$ E14-16) } & \multicolumn{2}{|c|}{ Terminal bud stage $(\sim \mathrm{E} 16-18)$} \\
\hline & & Early & Late & Early & Late \\
\hline Terminal bud & - & - & $+\mathrm{C},(\mathrm{M})$ & & \\
\hline Epithelial stalk & - & & & & \\
\hline Presumptive duct & & - & - & & \\
\hline Pro-acini & & & & $+\mathrm{C}, \mathrm{M}$ & $+\mathrm{A}, \mathrm{L}(\mathrm{C})$ \\
\hline Juxta-acinar cells & & & & $+\mathrm{A}$ & $+\mathrm{A}$ \\
\hline Intralobular duct & & & & - & $+\mathrm{A}$ \\
\hline Interlobular duct & & & & - & - \\
\hline
\end{tabular}

- negative staining, + positive staining, $C$ cytoplasmic staining, $M$ membrane staining, $A$ apical staining, $L$ lateral staining, () weak staining, empty slots: structure not present 
Fig. 1 AQP5 expression pattern during prenatal development of the mouse submandibular gland (SMG). A Pseudoglandular stage ( $\sim$ E14): no AQP5 staining is detectable in the terminal bud $(t)$, epithelial stalk $(e)$ or mesechyme $(m)$. B Early canalicular stage ( $\sim$ E14-15): AQP5 negative cells in the terminal bud $(t)$, presumptive duct $(p d)$, and mesenchyme $(m)$. C Late canalicular stage ( $\sim$ E15-E16): scattered positive pro-acinar cells are present (arrow). No AQP5 is found in the presumptive duct $(p d)$ or mesenchyme $(m)$. D Early terminal bud stage $(\sim$ E16E17): all pro-acinar cells are AQP5 positive (arrow). In the intralobular duct $(I A D)$, cells proximal to the pro-acini show apical AQP5 staining (arrowhead). E and F Late terminal bud stage $(\sim \mathrm{E} 17-18)$ : a similar expression pattern is observed as in D. Additionally, the rest of the intralobular duct (IAD) is also positive, and the interlobular duct (IED) is negative. Scale bar (A-E) $50 \mu \mathrm{m}$ and (F) $100 \mu \mathrm{m}$
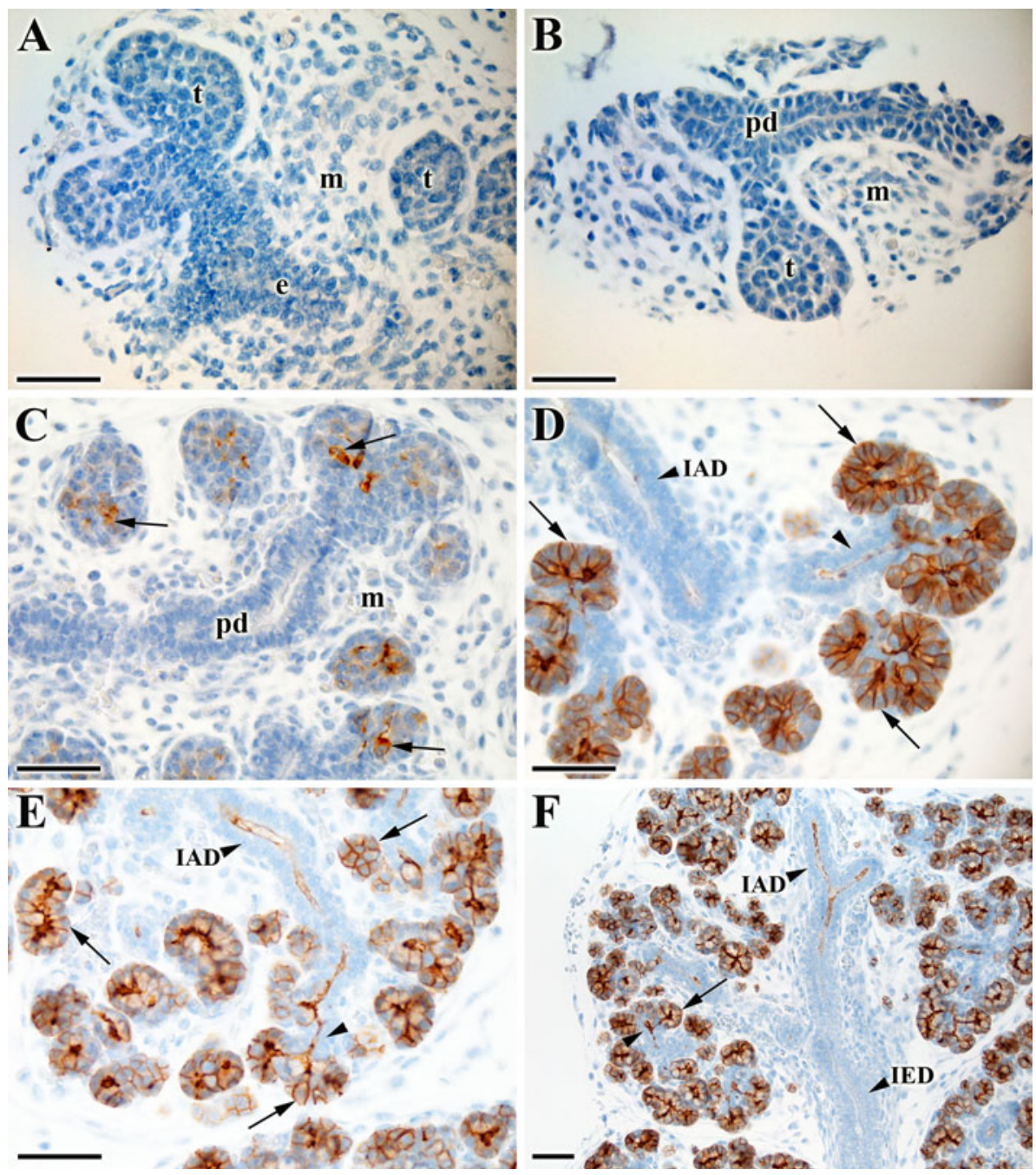

Table 2 AQP5 localization during postnatal development

\begin{tabular}{|c|c|c|c|c|c|c|c|}
\hline \multirow{2}{*}{+2} & \multirow{2}{*}{$\mathrm{P} 0$} & \multirow[t]{2}{*}{$\mathrm{P} 2$} & \multirow[t]{2}{*}{ P5 } & \multicolumn{2}{|l|}{$\mathrm{P} 25$} & \multicolumn{2}{|l|}{$\mathrm{P} 60$} \\
\hline & & & & Female & Male & Female & Male \\
\hline Pro-acini & $+\mathrm{A}, \mathrm{L}(\mathrm{B}, \mathrm{C})$ & $+\mathrm{A}, \mathrm{L}(\mathrm{B}, \mathrm{C})$ & $+\mathrm{A}, \mathrm{L}(\mathrm{B}, \mathrm{C})$ & & & & \\
\hline Acini & & & & $+\mathrm{A}, \mathrm{L}(\mathrm{B}, \mathrm{C})$ & $+\mathrm{A}, \mathrm{L}(\mathrm{B}, \mathrm{C})$ & $+\mathrm{A}, \mathrm{L}, \mathrm{B}$ & $+\mathrm{A}, \mathrm{L}, \mathrm{B}$ \\
\hline Intercalated duct & $+\mathrm{A}$ & $+\mathrm{A}$ & $+\mathrm{A}$ & $+\mathrm{A}^{*}$ & $+\mathrm{A}^{*}$ & $+\mathrm{A}^{*}$ & $+\mathrm{A}$ \\
\hline Intralobular duct & - & - & & & & & \\
\hline GCT & & & & - & - & - & - \\
\hline Striated duct & & & - & - & - & - & - \\
\hline Interlobular duct & - & - & - & - & - & - & - \\
\hline Excretory duct & & & - & - & - & - & - \\
\hline
\end{tabular}

+ positive staining, $A$ apical staining, $L$ lateral staining, $B$ basal staining, $C$ cytoplasmic staining, $\mathrm{A}^{*}$ apical staining only closest to the acini, negative staining, () weak staining, empty slots: structure not present

(P25), where the pro-acinar cells had developed into mature acinar cells, AQP5 was clearly localized to the apical and lateral membrane. Weaker staining was detected in the basal membrane and in the cytoplasm (Fig. 2C). The
ID was more convoluted and branched, making the transition between acini to ID more difficult to observe than in less mature glands. Nonetheless, in both sexes, the area proximal to the acini showed apical staining, while the 


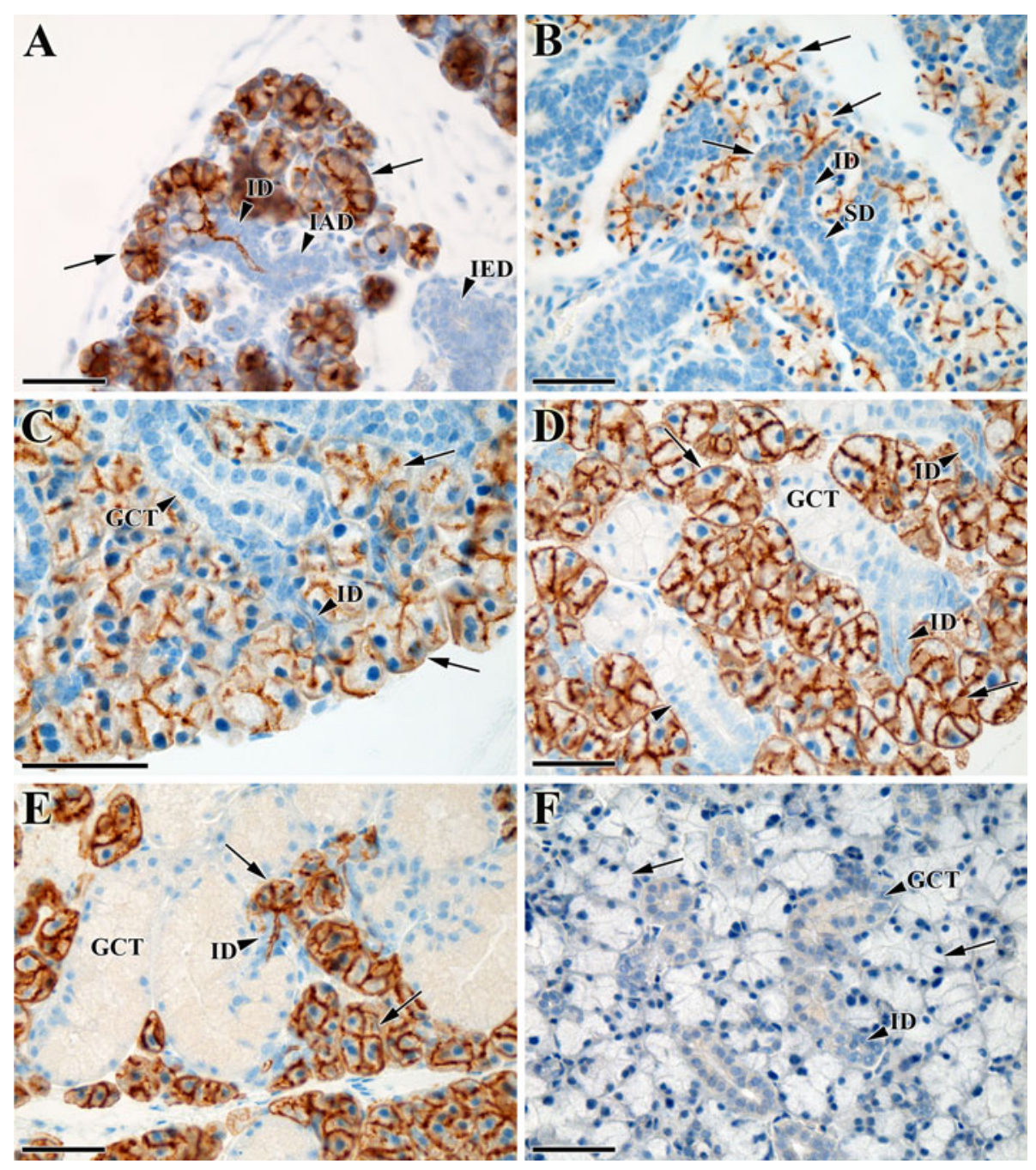

Fig. 2 AQP5 expression pattern during postnatal development of the mouse submandibular gland (SMG). A At birth (postnatal day 0, P0), the pro-acinar cells (arrow) and the intercalated ducts (ID) are AQP5 positive. No AQP5 is detected in the intralobular duct $(I A D)$ or interlobular duct (IED). B Pre-weaning (P5), both pro-acini (arrow) and intercalated ducts (ID) are AQP5 positive. No AQP5 is detected in the striated duct $(S D)$. C Young adult females (P25): acini (arrow) and the proximal part of the intercalated duct $(I D)$ are positive, while the granulated convoluted tubule $(G C T)$ is negative. D Adult females

distal part was negative. The following ductal segment had developed into the GCT, which could be recognized as larger cells with central nuclei. These were negative for AQP5 (Fig. 2C), as seen for all other ductal segments. In the adult gland of both female and male animals at P60, AQP5 could be detected in the luminal, lateral, and basal membrane of the acinar cells (Fig. 2D, E). At this age, sexual dimorphism is further developed in the gland (Gresik and Macrae 1975) and was evident when compared to glands from younger animals. Unlike what was observed in glands of young adults, AQP5 localized in the ID was found to be expressed in a gender specific manner at P60. While the ID of the female gland was, as shown at
(P60) show the same AQP5 pattern as in C. In addition, no AQP5 is detected in the transition from GCT to striated duct (arrowhead). E Adult males (P60): acini (arrow) and entire intercalated ducts (ID) are positive while the granulated convoluted tubule $(G C T)$ is negative. F IgG negative control in young adult female (P25) tissue shows no unspecific staining in the acini (arrow), intercalated duct (ID), or in the granulated convoluted tubule $(G C T)$. Granules in the granulated convoluted tubule are not seen using this method. A-F Scale bar $50 \mu \mathrm{m}$

$\mathrm{P} 25$, only positive in the part proximal to the acini (Fig. 2D), the male ID showed apical staining throughout the segment (Fig. 2E). The AQP5 negative GCT segment is at this age further matured, and the sexual dimorphism was more apparent. The cells were larger than in younger glands, and the nuclei were now basally placed (Fig. 2D, E). The remaining duct segments were also negative in both sexes. Immunostaining of embryonic kidney tissue, which expresses AQP1, 2, 3, 4, 7, and 11 but not AQP5, showed no reactivity, as expected (data not shown). Additionally, immunostaining of isotyped-matched negative control showed no staining in any region of SMG tissue (Fig. 2F). 
To examine the subcellular localization pattern of AQP5 in adult tissues that had not been subjected to embedding, confocal microscopy was used to compare AQP5 with other proteins of known localization. A cytosolic scaffolding component of tight junctions (Baker 2010), ZO1, has been detected in the apical-lateral region in a spider like distribution pattern in mature SMG acini of humans and mice (Kriegs et al. 2007; Larina and Thorn 2005; Maria et al. 2008). Comparison of AQP5 with ZO1 confirmed the luminal membrane localization of AQP5 (Fig. 3A, B, C). The co-localization of AQP5 with E-cadherin, a cell-cell adhesion protein found in lateral membranes of both acini and GCTs (Actis et al. 2002), confirmed a lateral localization of AQP5 (Fig. 3D, E, F). Also evident from these images was the lack of AQP5 expression within GCTs, which were classified on the basis of their distinct morphology (Fig. 3D, E, F). Immunostaining of collagen type IV (coll IV) was used to detect the basement membrane (Fig. 3G). The merged image of AQP5 and coll IV (Fig. 3I) demonstrated the localization of AQP5 within the basal cell membrane, detected as a signal apical to the basement membrane (Fig. 3G, H, I). Preabsorption of the antibody using its cognate peptide completely eliminated the apical, lateral, and basal expression of AQP5 (Fig. 3J, K). Also, comparison of the AQP5 antibody staining pattern with that of another antiAQP5 antibody, showed similar results (data not shown).

To confirm the localization of AQP5 within the different regions of the acinar plasma membrane at higher resolution, both male and female P60 glands were immunogold labeled and analyzed using TEM. AQP5 was localized to the luminal membranes, including the laterally placed intercellular canaliculi and the apical membrane portion (Fig. 4A, B, C). Some staining was also found in the remaining portion of the interdigitated lateral membrane facing the neighboring cell (Fig. 4A). In basal regions, AQP5 was especially observed in areas with digits; mainly found between adjacent cells (Fig. 4A, D). There was also some staining scattered in the ER. AQP5 negative myoepithelial cells could be found in between the acinar cells' basal membrane and the basal lamina. The acinar basal membrane adjoining the myoepithelial cells was mostly negative, but did have some staining where digits were present. The general acinar AQP5 localization pattern was indistinguishable in male and female SMG tissues.

\section{Discussion}

The present study revealed the temporal-spatial AQP5 expression pattern during mouse SMG development. AQP5 was primarily distributed in the secretory portion of the gland, with some variation in ductal localization.
Interestingly, positive AQP5 staining in the entire IAD was found in the late terminal bud stage. Also, a gender difference was apparent in the adult gland ID but not in acinar cells.

The appearance of AQP5 in the late canalicular stage confirms previous reports where it was first detected in late embryonic development (Abate et al. 2003; Larsen et al. 2009). In the early terminal bud stage, where cytodifferentiation is well on its way, all pro-acinar cells were positive and had strong cytoplasmic staining. Intracellular localization of AQP5 has also been detected at a comparable stage of development in the rat SMG (Akamatsu et al. 2003). In addition, AQP5 membrane staining was more apparent at this stage compared to the previous one. One could speculate that due to the increased AQP5 expression in the membranes, the cytoplasmic distribution represents AQP5 proteins being processed in the ER and Golgi apparatus. An earlier study using organ culture indicated that lumen formation and cell polarization indirectly regulate the AQP5 polarized membrane localization (Hashizume and Hieda 2006). Thus, the modification of AQP5 localization during embryonic development seen in our results may reflect cell polarization and lumen formation in the terminal buds.

In the postnatal gland, AQP5 plays a significant functional role in salivary fluid secretion (Kawedia et al. 2007; Krane et al. 2001; Ma et al. 1999). In the adult mouse SMG, AQP5 has previously been reported to be localized to the luminal membrane, apical pole, or apical region of acinar cells (Larina and Thorn 2005; Ma et al. 1999; Nandula et al. 2007). The present study; however, depicts a more nuanced pattern of AQP5 in pro-acinar/acinar cells with not only luminal (apical and canalicular), but also lateral and an increasingly prominent basal membrane localization. Although localization of AQP5 in the basal membrane has not previously been found in the mouse SMG, studies from both the mouse parotid gland and the rat SMG have shown similar results (Matsuzaki et al. 2006; Murdiastuti et al. 2002). It has been proposed that basally placed AQP5 in the rat SMG may act as an osmosensor, thereby regulating paracellular fluid transfer (Murakami et al. 2006). In the mouse parotid gland, transcellular and paracellular fluid transport work in conjunction, and an association of AQP5 and tight junction complexes seems to play a role in this process (Kawedia et al. 2007). The relationship between AQP5 and tight junctions in the mouse SMG has not been specifically examined, but our results do demonstrate a close association between the two proteins.

Although a previous report showed low levels of AQP5 in the developing ducts as early as E15.5 (Walker et al. 2008), we could not confirm this finding in the present study. AQP5 was, however, found in the apical membrane 

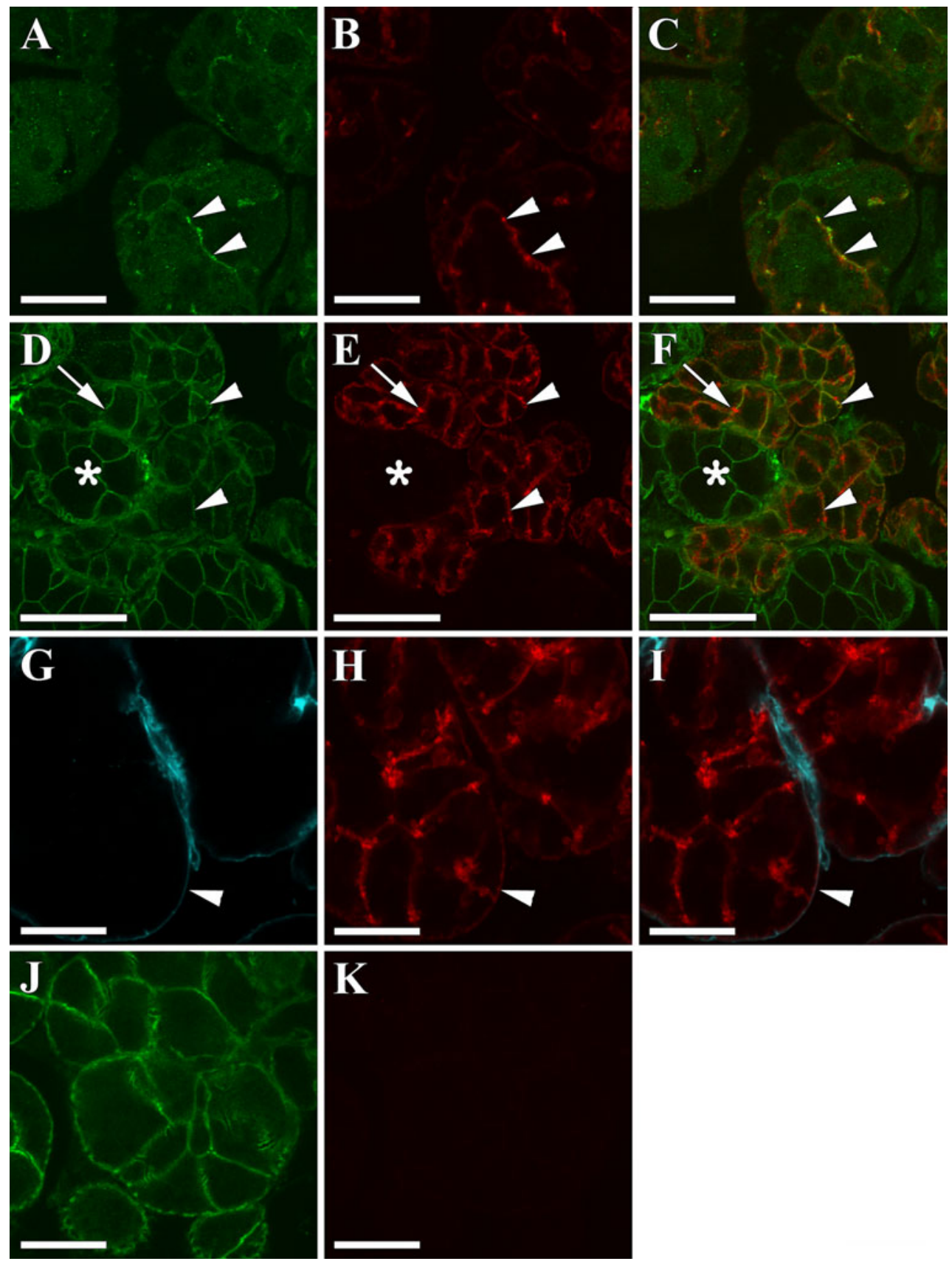

Fig. 3 Confocal imaging of the adult SMG (P60). Aquaporin 5 (AQP5) demonstrates an apical, lateral, and basal localization in the SMG acini. A Localization of ZO-1 (green) near luminal membranes of acinar cells. B Localization of AQP5 (red) within the same cells as in A. C Merged image of $\mathbf{A}$ and $\mathbf{B}$ confirms the apical localization of AQP5. Arrow heads luminal membrane (scale bar $20 \mu \mathrm{m}$ ). D Localization of E-cadherin (green) within lateral membranes in both acinar (arrow heads) and GCT cells (*). E Localization of AQP5 (red) in the acinar cells. F Merged image of $\mathbf{D}$ and $\mathbf{E}$ demonstrates a lateral localization of AQP5 (yellow/orange), designated by arrowheads. An AQP5 and E-cadherin non-overlapping pattern of expression in the

of juxta-acinar cells from the early terminal bud stage, and this pattern was consistent towards birth and during postnatal development. Surprisingly, the entire IAD showed luminal canaliculi is evident (arrow). Non-overlapping patterns are also seen in the GCT cells (*) (scale bar, $50 \mu \mathrm{m}$ ). G Immunostaining of the basement membrane protein coll IV (blue). H Localization of AQP5 (red) in the acinar cells. I Merged image confirms a basal localization (arrow head) of AQP5 distinct from coll IV localization (scale bar $20 \mu \mathrm{m}$ ). J Localization of E-cadherin (green) within lateral membranes in acinar cells in a tissue immunostained with preabsorbed AQP5. K Preabsorption of the AQP5 antibody using its cognate peptide, showing complete elimination of the AQP5 staining pattern in the same cells as in $\mathbf{J}$ (scale bar $20 \mu \mathrm{m}$ ). A-I images are of male P60, J-K images are of female P60

extensive, stage-specific AQP5 distribution in the late terminal bud stage, just prior to birth. This finding has, to our knowledge, never been described, although a previous 
Fig. 4 Gold labeling of AQP5 in the SMG of adult animals (P60). A Overview of AQP5 gold staining in the basal membrane, membranes of intercellular canaliculi, as well as the lateral membrane $(L M)$. $C L$ canalicular lumen, $B D$ basal digits (scale bar, $1 \mu \mathrm{m}$ ). B Gold labeling of AQP5 was detected in the apical membrane of acinar cells (scale bar, $1 \mu \mathrm{m}$ ). C Longitudinal section of an intercellular canaliculus showing gold labeling of AQP5 in the membrane (scale bar, $0.5 \mu \mathrm{m}$ ). D AQP5 was localized in the basal membrane in areas where digits could be seen, while no AQP5 was detectable in the basement membrane (scale bar $0.2 \mu \mathrm{m}$ ). Non-linear adjustments were applied to entire images

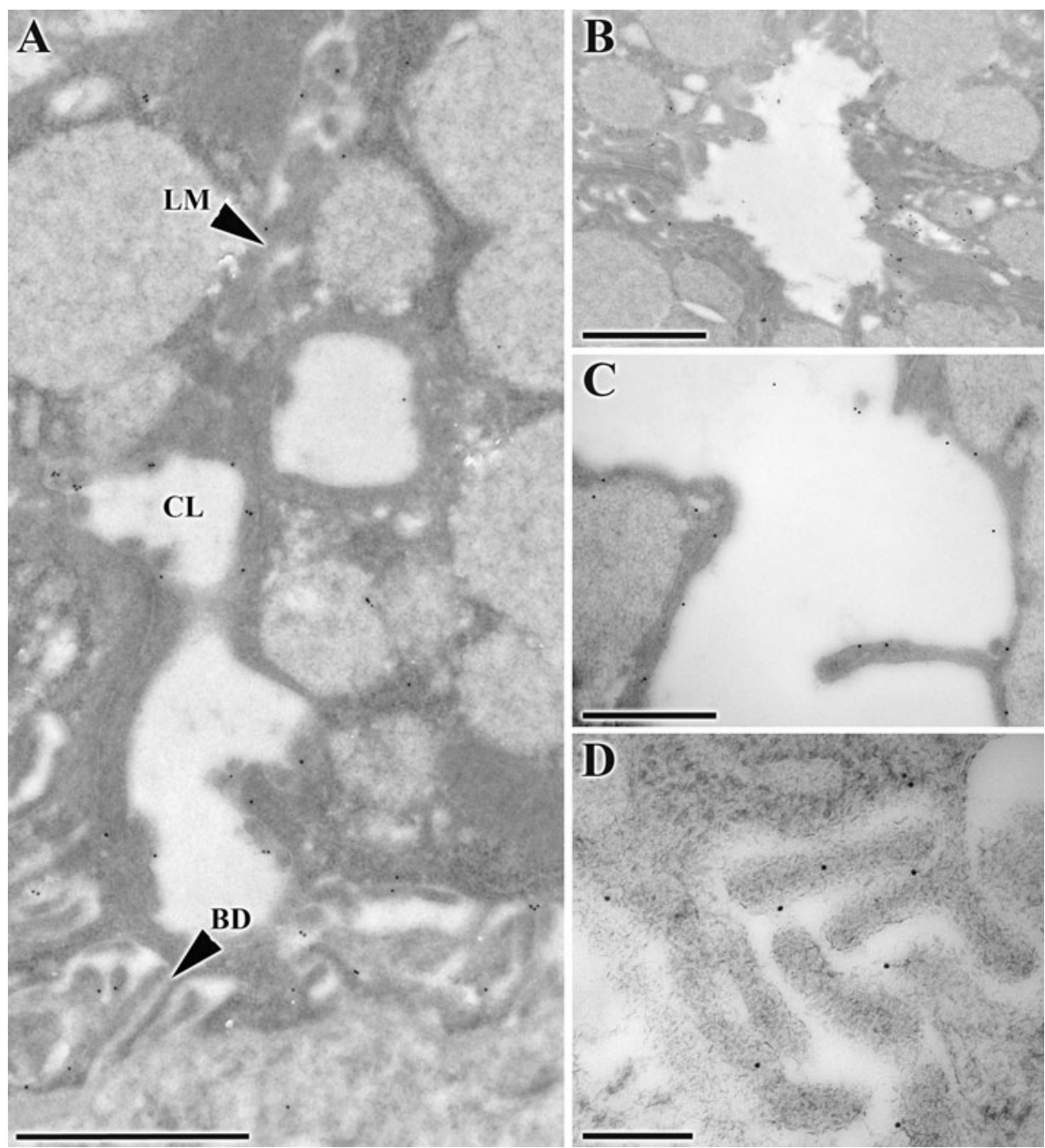

study identified increased AQP5 protein expression levels during the same period by Western analysis (Larsen et al. 2009). The reason for this transient AQP5 increase is not known, but might point to a novel developmental role in addition to its well known importance in salivary fluid secretion.

Sexual dimorphism in the mouse SMG is not present at birth, but develops postnatally and is previously reported to be evident by young adult stages (Gresik and Macrae 1975). Previous studies have shown that in the ID segment there are sexual differences with regards to both cell morphology and physiology (Chai et al. 1993; Denny and Denny 1999; Gresik and Macrae 1975). Based on morphology, the segment can be divided into two portions during postnatal development: a granulated and a nongranulated portion found in females, while only the latter is found in males (Caramia 1966; Gresik and Macrae 1975). It might be speculated that AQP5 is associated with the granulated portions of the ID in the female gland. However, such an association does not explain the presence of AQP5 in the entire male ID, which consists of non-granulated cells. At P25 sexual dimorphism in the ID segment is not yet fully developed (Gresik and Macrae 1975), which is consistent with the AQP5 distribution reported here. The localization of AQP5 in the ID of rat SMG has previously been found by some (Gresz et al. 2004; Matsuzaki et al. 1999), but has not been reported in the mouse SMG. Further studies are needed to fully understand the age and gender specific role of AQP5 in the ID.

In this investigation we have evaluated AQP5 expression at all stages of embryonic development and at various adult stages. Additionally, we have used three different techniques to verify the AQP5 localization in the adult SMG. AQP5 is widely used as an apical marker in various mouse SMG experiments, ranging from embryonic to adult SMGs. It is, therefore, important to know its specific distribution throughout gland development. This study describes AQP5 as first appearing well after the initial branching period with a relatively stable expression pattern confined to the terminal tubuli/pro-acinar and the juxtaacinar cells throughout embryonic development. In the postnatal gland, a general pattern is observed where the ID 
and pro-acini/acini express AQP5. Taken together, the expression pattern of AQP5 is shown to reflect the complexity of SMG development by demonstrating an evolving distribution throughout glandular growth and maturation.

Acknowledgments This work has been funded by the Faculty of Dentistry, University of Oslo, Norway, the NIH (RC1DE02040201, and R21DE019197 to M. L), and the Norwegian Association of Higher Education Institutions. We are most thankful for the protein A gold and expert advice from Dr. Sverre Henning Brorson at the Institute of Pathology at the University of Oslo. We would also like to thank Olav J. F. Schreurs, Hanne Kristiansen, Yiqing Cai, and Benedicto Abrigos Geronimo at the Department of Oral Biology, University of Oslo for valuable technical assistance. The authors declare that they have no financial conflicts of interest.

Open Access This article is distributed under the terms of the Creative Commons Attribution Noncommercial License which permits any noncommercial use, distribution, and reproduction in any medium, provided the original author(s) and source are credited.

\section{References}

Abate E, Krane C, Rizvi T, Colbert M, Jiang M, Boivin G, Menon A (2003) Tissue distribution of aquaporin 5 in the developing salivary gland and lung of mouse embryo. J Invest Med 51:S143

Actis AB, Lampe PD, Eynard AR (2002) Cellular basis and clinical implications of biological markers in salivary tissues: their topological distribution in murine submandibular gland. Oral Oncol 38:441-449. doi:10.1016/S1368-8375(01)00091-4

Akamatsu T, Parvin MN, Murdiastuti K, Kosugi-Tanaka C, Yao CJ, Miki O, Kanamori N, Hosoi K (2003) Expression and localization of aquaporins, members of the water channel family, during development of the rat submandibular gland. Pflugers Arch-Euro J Physiol 446:641-651

Baker OJ (2010) Tight junctions in salivary epithelium. J Biomed Biotech 2010:13. doi:10.1155/2010/278948

Caramia F (1966) Ultrastructure of mouse submaxillary gland. I. Sexual differences. J Ultrastruct Res 16:505-523

Chai Y, Klauser DK, Denny PA, Denny PC (1993) Proliferative and structural differences between male and female mouse submandibular glands. Anat Rec 235:303-311

Daley WP, Gulfo KM, Sequeira SJ, Larsen M (2009) Identification of a mechanochemical checkpoint and negative feedback loop regulating branching morphogenesis. Dev Biol 336:169-182. doi:10.1016/j.ydbio.2009.09.037

Denny PC, Denny PA (1999) Dynamics of parenchymal cell division, differentiation, and apoptosis in the young adult female mouse submandibular gland. Anat Rec 254:408-417. doi:10.1002/ (SICI)1097-0185(19990301)254:3<408:AID-AR12>3.0.CO;2-G

Denny PA, Pimprapaiporn W, Kim MS, Denny PC (1988) Quantitation and localization of acinar cell-specific mucin in submandibular glands of mice during postnatal-development. Cell Tiss Res 251:381-386. doi:10.1007/BF00215847

Gresik EW, Macrae EK (1975) The postnatal development of the sexually dimorphic duct system and of amylase activity in submandibular glands of mice. Cell Tiss Res 157:411-422. doi: $10.1007 / \mathrm{BF} 00225529$

Gresz V, Kwon TH, Hurley PT, Varga G, Zelles T, Nielsen S, Case RM, Steward MC (2001) Identification and localization of aquaporin water channels in human salivary glands. Am J Physiol-Gastroint Liver Physiol 281:G247-G254
Gresz V, Kwon TH, Gong H, Agre P, Steward MC, King LS, Nielsen $S$ (2004) Immunolocalization of AQP-5 in rat parotid and submandibular salivary glands after stimulation or inhibition of secretion in vivo. Am J Physiol-Gastroint Liver Physiol 287:G151-G161. doi:10.1152/ajpgi.00480.2003

Hashizume A, Hieda Y (2006) Hedgehog peptide promotes cell polarization and lumen formation in developing mouse submandibular gland. Biochem Biophys Res Comm 339:996-1000. doi:10.1016/j.bbrc.2005.11.106

Ishibashi K, Hara S, Kondo S (2009) Aquaporin water channels in mammals. Clin Exp Nephrol 13:107-117. doi:10.1007/s10157008-0118-6

Jaskoll T, Melnick M (1999) Submandibular gland morphogenesis: stage-specific expression of TGF-alpha/EGF, IGF, TGF-beta, TNF, and IL-6 signal transduction in normal embryonic mice and the phenotypic effects of TGF-beta 2, TGF-beta 3, and EGF$\mathrm{R}$ null mutations. Anatom Rec 256:252-268. doi:10.1002/ (SICI)1097-0185(19991101)256:3<252:AID-AR5>3.0.CO;2-6

Kawedia JD, Nieman ML, Boivin GP, Melvin JE, Kikuchi KI, Hand AR, Lorenz JN, Menon AG (2007) Interaction between transcellular and paracellular water transport pathways through aquaporin 5 and the tight junction complex. Proc Natl Acad Sci U S A 104:3621-3626. doi:10.1073/pnas.0608384104

Krane CM, Goldstein DL (2007) Comparative functional analysis of aquaporins/glyceroporins in mammals and anurans. Mamm Gen 18:452-462. doi:10.1007/s00335-007-9041-5

Krane CM, Melvin JE, Nguyen HV, Richardson L, Towne JE, Doetschman T, Menon AG (2001) Salivary acinar cells from aquaporin 5-deficient mice have decreased membrane water permeability and altered cell volume regulation. J Biol Chem 276:23413-23420. doi:10.1074/jbc.M008760200

Kriegs JO, Homann V, Kinne-Saffran E, Kinne RKH (2007) Identification and subcellular localization of paracellin-1 (claudin-16) in human salivary glands. Histochem Cell Biol 128:45-53. doi:10.1007/s00418-007-0291-9

Larina O, Thorn P (2005) Ca2+ dynamics in salivary acinar cells: distinct morphology of the acinar lumen underlies near-synchronous global $\mathrm{Ca} 2+$ responses. J Cell Sci 118:4131-4139. doi: $10.1242 /$ jcs. 02533

Larsen M, Hoffman MP, Sakai T, Neibaur JC, Mitchell JM, Yamada KM (2003) Role of PI 3-kinase and PIP3 in submandibular gland branching morphogenesis. Dev Biol 255:178-191. doi:10.1016/ S0012-1606(02)00047-7

Larsen HS, Ruus AK, Galtung HK (2009) Aquaporin expression patterns in the developing mouse salivary gland. Europ J Oral Sci 117:655-662. doi:10.1111/j.1600-0722.2009.00695.x

Larsen HS, Ruus AK, Schreurs O, Galtung HK (2010) Aquaporin 11 in the developing mouse submandibular gland. Eur J Oral Sci 118:9-13. doi:10.1111/j.1600-0722.2009.00708.x

Ma T, Song Y, Gillespie A, Carlson EJ, Epstein CJ, Verkman AS (1999) Defective secretion of saliva in transgenic mice lacking aquaporin-5 water channels. J Biol Chem 274:20071-20074. doi: 10.1074/jbc.274.29.20071

Maria OM, Kim JWM, Gerstenhaber JA, Baum BJ, Tran SD (2008) Distribution of tight junction proteins in adult human salivary glands. J Histochem Cytochem 56:1093-1098. doi:10.1369/jhc.2008.951780

Matsuzaki T, Suzuki T, Koyama H, Tanaka S, Takata K (1999) Aquaporin-5 (AQP5), a water channel protein, in the rat salivary and lacrimal glands: immunolocalization and effect of secretory stimulation. Cell Tissue Res 295:513-521. doi:10.1007/ s004410051257

Matsuzaki T, Ablimit A, Suzuki T, Aoki T, Hagiwara H, Takata K (2006) Changes of aquaporin 5-distribution during release and reaccumulation of secretory granules in isoproterenol-treated mouse parotid gland. J Electron Microsc 55:183-189. doi: 10.1093/jmicro/dfl023 
Murakami M, Murdiastuti K, Hosoi K, Hill AE (2006) AQP and the control of fluid transport in a salivary gland. J Membr Biol 210:91-103. doi:10.1007/s00232-005-0848-2

Murdiastuti K, Miki O, Yao CJ, Parvin MN, Kosugi-Tanaka C, Akamatsu T, Kanamori N, Hosoi K (2002) Divergent expression and localization of aquaporin 5, an exocrine-type water channel, in the submandibular gland of Sprague-Dawley rats. Pflugers Arch-Europ J Physiol 445:405-412. doi:10.1007/s00424-0020940-8

Nandula SR, Amarnath S, Molinolo A, Bandyopadhyay BC, Hall B, Goldsmith CM, Zheng CY, Larsson J, Sreenath T, Chen WJ, Ambudkar IS, Karlsson S, Baum BJ, Kulkarni AB (2007) Female mice are more susceptible to developing inflammatory disorders due to impaired transforming growth factor beta signaling in salivary glands. Arthritis Rheumat 56:1798-1805. doi:10.1002/art.22715

Parreira KS, Debaix H, Cnops Y, Geffers L, Devuyst O (2009) Expression patterns of the aquaporin gene family during renal development: influence of genetic variability. Pflugers Arch Europ J Physiol 458:745-759. doi:10.1007/s00424-009-0667-x

Tucker AS (2007) Salivary gland development. Sem Cell Develop Biol 18:237-244. doi:10.1016/j.semcdb.2007.01.006

Walker JL, Menko AS, Khalil S, Rebustini I, Hofftnan MP, Kreidberg JA, Kukuruzinska MA (2008) Diverse roles of E-cadherin in the morphogenesis of the submandibular gland: insights into the formation of acinar and ductal structures. Develop Dynam 237:3128-3141. doi:10.1002/dvdy.21717

Wei C, Larsen M, Hoffman MP, Yamada KM (2007) Self-organization and branching morphogenesis of primary salivary epithelial cells. Tissue Eng 13:721-735. doi:10.1089/ten.2006.0123

Young JA, Van Lennep EW (1978) The morphology of salivary glands. Academic Press Inc, London

Zinzen KM, Hand AR, Yankova M, Ball WD, Mirels L (2004) Molecular cloning and characterization of the neonatal rat and mouse submandibular gland protein SMGC. Gene 334:23-33. doi:10.1016/j.gene.2004.03.014 